\title{
Phytoprotection
}

\section{Comparaison de techniques d'identification des Erwinia et des Pseudomonas responsables de la pourriture molle}

\section{Lacroix, L. Vézina, S. Desjardins et C. Beaulieu}

Volume 76, numéro 1, 1995

URI : https://id.erudit.org/iderudit/706082ar

DOI : https://doi.org/10.7202/706082ar

Aller au sommaire du numéro

Éditeur(s)

Société de protection des plantes du Québec (SPPQ)l

ISSN

0031-9511 (imprimé)

1710-1603 (numérique)

Découvrir la revue

Citer cet article

Lacroix, M., Vézina, L., Desjardins, S. \& Beaulieu, C. (1995). Comparaison de techniques d'identification des Erwinia et des Pseudomonas responsables de la pourriture molle. Phytoprotection, 76(1), 27-37.

https://doi.org/10.7202/706082ar
Résumé de l'article

Trois méthodes, soit la caractérisation physiologique, l'utilisation de systèmes miniaturisés d'identification (API 20E, API NFT et Biolog) et l'analyse du profil électrophorétique des protéines sécrétées, ont été expérimentées afin de déterminer une technique précise et rapide d'identification des Pseudomonas et des Erwinia responsables de la pourriture molle. L'analyse des patrons électrophorétiques des protéines sécrétées est une méthode très efficace pour identifier les différentes espèces pectinolytiques de Pseudomonas fluorescents. Le système Biolog reconnaît efficacement le $P$. marginalis et le $P$. viridiflava. Le système API NFT est efficace pour l'identification du $P$. marginalis, du $P$. viridiflava et du $P$. syringae. C'est le système API 20E qui s'est avéré le plus efficace pour l'identification des Erwinia. L'électrophorèse des protéines sécrétées et le système API NFT permettent une identification rapide et efficace des Pseudomonas, tandis que pour les Erwinia, seul le système API20E est performant. 


\title{
Comparaison de techniques d'identification des Erwinia et des Pseudomonas responsables de la pourriture molle
}

\author{
Michel Lacroix ${ }^{1}$, Lise Vézina ${ }^{1}$, Suzanne Desjardins ${ }^{1}$ et Carole \\ Beaulieu ${ }^{2}$
}

Reçu 1994-07-07; accepté 1994-12-15

Trois méthodes, soit la caractérisation physiologique, I'utilisation de systèmes miniaturisés d'identification (API 20E, API NFT et Biolog) et l'analyse du profil électrophorétique des protéines sécrétées, ont été expérimentées afin de déterminer une technique précise et rapide d'identification des Pseudomonas et des Erwinia responsables de la pourriture molle. L'analyse des patrons électrophorétiques des protéines sécrétées est une méthode très efficace pour identifier les différentes espèces pectinolytiques de Pseudomonas fluorescents. Le système Biolog reconnaît efficacement le $P$. marginalis et le $P$. viridiflava. Le système API NFT est efficace pour l'identification du $P$. marginalis, du $P$. viridiflava et du $P$. syringae. C'est le système API 20E qui s'est avéré le plus efficace pour l'identification des Erwinia. L'électrophorèse des protéines sécrétées et le système API NFT permettent une identification rapide et efficace des Pseudomonas, tandis que pour les Erwinia, seul le système API 20E est performant.

Lacroix, M., L. Vézina, S. Desjardins, and C. Beaulieu. 1995. Comparison of identification methods for Erwinia and Pseudomonas species causing soft rot diseases. PHYTOPROTECTION 76 : 27-37.

Three methods, namely physiological characterization, the use of miniaturized identification systems (API 20E, API NFT, and Biolog) and the analysis of the electrophoretic profile of proteins secreted by the bacteria, were used in order to determine a precise and quick identification technique for the Pseudomonas and Erwinia species causing soft rots in several plant species. The analysis of the electrophoretic profile of the proteins secreted is a very efficient method of identification for the various pectinolytic species of fluorescent Pseudomonas. The Biolog system accurately recognizes $P$. marginalis and $P$. viridiflava. The API NFT system is efficient for the identification of $P$. marginalis, $P$. viridiflava and $P$. syringae. The API $20 \mathrm{E}$ system was found to be the most reliable system for identifying Erwinia species. The electrophoresis of proteins secreted and the API NFT system allow a quick and efficient identification of Pseudomonas species, while for Erwinia species, only the API 20E system is efficient.

1. Service de phytotechnie de Québec, Ministère de l'Agriculture, des Pêcheries et de I'Alimentation du Québec, Complexe scientifique, 2700, rue Einstein, Sainte-Foy (Québec), Canada G1P 3W8

2. Département de biologie, Université de Sherbrooke, Sherbrooke (Québec), Canada J1K 2R1 


\section{INTRODUCTION}

Le laboratoire de diagnostic du ministère de I'Agriculture, des Pêcheries et de I'Alimentation du Québec (MAPAQ) reçoit chaque année de nombreuses plantes cultivées atteintes de la pourriture molle. Entre 1989 et 1993, la pourriture molle a été la maladie bactérienne la plus souvent diagnostiquée, avec 216 diagnostics positifs sur 561 échantillons ayant des symptômes causés par des bactéries. Les agents bactériens responsables de la pourriture molle appartiennent à plusieurs genres: Erwinia, Pseudomonas, Bacillus, Clostridium, Flavobacterium, Cytophaga et Xanthomonas (Liao et Wells 1987a; Lund 1983). Les bactéries pectinolytiques des genres Erwinia et Pseudomonas sont cependant les plus fréquentes en Amérique du Nord (Cappellini et al. 1987; Ceponis et al. 1985; Gitaitis et al. 1991; Hildebrand 1989; Liao et Wells 1987b; Pieczarka et Lorbeer 1975).

Différentes méthodes ont été utilisées pour identifier les Erwinia et les Pseudomonas pectinolytiques : la caractérisation des bactéries selon des critères physiologiques (Dickey et Kelman 1988; Hildebrand et al. 1971; Lelliott et al. 1966), I'analyse sérologique (Dickey et al. 1984; Samson et al. 1987), I'hybridation ADNADN (Palleroni 1984), la comparaison des profils protéiques (Van Zyl et Steyn 1990), le polymorphisme des fragments de restriction de I'ADN (RFLP) (Boccara et al. 1991; Ward et De Boer 1994) et l'amplification en chaîne par polymérase (PCR) (Darrasse et al. 1994). Plusieurs de ces techniques sont laborieuses, coûteuses ou requièrent de l'équipement spécialisé. Certaines compagnies offrent donc aux laboratoires faisant de l'identification bactérienne de façon routinière, des systèmes miniaturisés d'identification (API 20E, API NFT, Biolog, etc.) qui permettent d'évaluer rapidement plusieurs propriétés physiologiques et biochimiques des bactéries.

Au laboratoire de diagnostic du MAPAQ, les bactéries pectinolytiques des genres Pseudomonas fluorescents et Erwinia sont identifiées de façon routinière selon les critères physiologiques proposés par Dickey et Kelman (1988), et par Lelliott et al. (1966). Ce travail compare l'efficacité de différents systèmes (API 20E, API NFT, Biolog) à identifier les bactéries pectinolytiques des genres Pseudomonas fluorescents et Erwinia. L'analyse des profils protéiques est une excellente méthode pour différencier les sousespèces ou les pathovars de différentes espèces de bactéries phytopathogènes (Paradis etal. 1994; Van Zyl et Steyn 1990). Toutefois, comme cette méthode requiert de nombreuses étapes de manipulation, telles que lyse des cellules, extraction et dosage des protéines, électrophorèse, lecture etcomparaison des profils électrophorétiques, il devient difficile de l'utiliser de façon routinière. Nous avons plutôt comparé le profil électrophorétique des protéines sécrétées par les bactéries de la pourriture molle. Cette méthode aurait l'avantage d'éliminer les étapes de: lyse cellulaire et d'extraction protéique.

\section{MATÉRIEL ET MÉTHODES}

\section{Isolement des bactéries pectinolytiques}

Les bactéries ont été isolées des tissus situés immédiatement sous la zone de pourriture molle. Un morceau de tissu d'environ $1 \mathrm{~cm}^{3}$ a été prélevé et déposé dans $2 \mathrm{~mL}$ d'eau physiologique $(\mathrm{NaCl}$ $0,85 \%)$ pendant $30 \mathrm{~min}$. À l'aide d'une micropipette, $100 \mu \mathrm{L}$ de cette eau de trempage a été prélevée et transférée dans des boîtes de Pétri contenant du milieu MS (Miller et Schroth 1972) ou du milieu $B$ de King (King et al. 1954). Les colonies présentant les caractéristiques des Erwinia (colonies orangées ayant une marge dentelée sur le milieu MS) ou des Pseudomonas (colonies produisant une fluorescence sur le milieu B de King) ont été purifiées par des repiquages successifs sur des milieux de culture. La liste des bactéries utilisées dans ce travail est donnée au tableau 1.

\section{Caractérisation physiologique des bactéries}

Les souches de Pseudomonas fluorescents ont été identifiées au niveau de l'espèce selon les critères suivants: production de lévane (Lelliott et al. 1966), réaction d'oxydase (Lelliott et al. 1966), activité pectinolytique (Hildebrand 1971), production d'arginine déshydrolase et 
Tableau 1. Liste des souches bactériennes de Pseudomonas et d'Erwinia utilisées

\begin{tabular}{|c|c|c|c|}
\hline $\begin{array}{l}\text { Souche } \\
n^{\circ}\end{array}$ & $\begin{array}{l}\text { Identification par des } \\
\text { tests physiologiques }\end{array}$ & Isolée de & Provenance \\
\hline 51 & P. marginalis & Brassica oleracea var. italica & P.D. Hildebrand \\
\hline 94 & P. marginalis & Capsicum annuum var. annuum & MAPAQ $^{\mathrm{a}}$ \\
\hline 95 & P. marginalis & Capsicum annuum var. annuum & MAPAQ \\
\hline 101 & P. marginalis & Apium graveolens var. dulce & MAPAQ \\
\hline 114 & P. marginalis & Solanum tuberosum & MAPAQ \\
\hline 176 & P. marginalis & Allium cepa & MAPAQ \\
\hline 192 & P. marginalis & Allium cepa & MAPAQ \\
\hline 194 & P. marginalis & Allium cepa & MAPAQ \\
\hline 215 & P. marginalis & Aconitum sp. & MAPAQ \\
\hline 221 & P. marginalis & Aconitum sp. & MAPAQ \\
\hline 292 & P. marginalis & Lactuca sativa & MAPAQ \\
\hline 357 & P. marginalis & Lycopersicon esculentum & MAPAQ \\
\hline 58 & P. viridiflava & Brassica oleracea var. gemmifera & P.D. Hildebrand \\
\hline 59 & P. viridiflava & Brassica oleracea var. gemmifera & P.D. Hildebrand \\
\hline 78 & $P$. viridiflava & Chrysanthemum sp. & J.F. Chauveau \\
\hline 183 & P. viridiflava & Solanum melongena var. esculentum & MAPAO \\
\hline 97 & P. syringae & Capsicum annuum var. annuum & MAPAQ \\
\hline 135 & P. syringae & Brassica oleracea var. botrytis & MAPAO \\
\hline 136 & P. syringae & Brassica oleracea var. botrytis & MAPAQ \\
\hline 137 & P. syringae & Brassica oleracea var. botrytis & MAPAO \\
\hline 57 & P. fluorescens & Brassica oleracea var. italica & P.D. Hildebrand \\
\hline 110 & P. fluorescens & Fœniculum sp. & MAPAQ \\
\hline 115 & P. fluorescens & Gerbera jamesonii & MAPAQ \\
\hline 120 & $P$. fluorescens & Brassica oleracea var. capitata & MAPAQ \\
\hline 129 & P. fluorescens & Solanum tuberosum & MAPAQ \\
\hline 142 & $P$. fluorescens & Brassica campestris var. pekinensis & MAPAQ \\
\hline 296 & P. fluorescens & Lactuca sativa & MAPAQ \\
\hline 322 & P. fluorescens & Cucumis sativus & MAPAQ \\
\hline 326 & $P$. fluorescens & Cucumis sativus & MAPAQ \\
\hline 331 & P. fluorescens & Cucumis sativus & MAPAQ \\
\hline 13 & E. carotovora ssp. carotovora & Solanum tuberosum & ATCC15713 \\
\hline 15 & E. carotovora ssp. carotovora & Daucus carota var. sativus & ATCC25206 \\
\hline 16 & E. carotovora ssp. carotovora & Solanum tuberosum & ATCC25270 \\
\hline 74 & E. carotovora ssp. carotovora & Solanum tuberosum & J.F. Chauveau \\
\hline 130 & E. carotovora ssp. carotovora & Solanum tuberosum & MAPAO \\
\hline 138 & E. carotovora ssp. carotovora & Solanum tuberosum & MAPAQ \\
\hline 153 & E. carotovora ssp. carotovora & Solanum tuberosum & MAPAO \\
\hline 162 & E. carotovora ssp. carotovora & Schlumbergera sp. & MAPAQ \\
\hline 163 & E. carotovora ssp. carotovora & Schlumbergera sp. & MAPAQ \\
\hline 166 & E. carotovora ssp. carotovora & Schlumbergera sp. & MAPAQ \\
\hline 169 & E. carotovora ssp. carotovora & Schlumbergera sp. & MAPAQ \\
\hline 172 & E. carotovora ssp. carotovora & Lilium sp. & MAPAO \\
\hline 182 & E. carotovora ssp. carotovora & Euphorbia sp. & MAPAO \\
\hline 226 & E. carotovora ssp. carotovora & Cucumis sativus & MAPAQ \\
\hline 239 & E. carotovora ssp. carotovora & Solanum tuberosum & MAPAO \\
\hline 375 & E. carotovora ssp. carotovora & Lactuca sativa & MAPAO \\
\hline 382 & E. carotovora ssp. carotovora & Solanum tuberosum & MAPAQ \\
\hline 413 & E. carotovora ssp. carotovora & Solanum tuberosum & MAPAQ \\
\hline 22 & E. carotovora ssp. atroseptica & Solanum tuberosum & ATCC33260 \\
\hline 62 & E. carotovora ssp. atroseptica & Solanum tuberosum & MAPAQ \\
\hline 157 & E. carotovora ssp. atroseptica & Solanum tuberosum & MAPAO \\
\hline 690 & E. carotovora ssp. atroseptica & Solanum tuberosum & MAPAQ \\
\hline 707 & E. carotovora ssp. atroseptica & Solanum tuberosum & MAPAQ \\
\hline 749 & E. carotovora ssp. atroseptica & Solanum tuberosum & MAPAO \\
\hline
\end{tabular}

a Souche isolée et caractérisée au laboratoire de diagnostic du ministère de l'Agriculture, des Pêcheries et de l'Alimentation du Québec. 
hypersensibilité sur tabac (Lelliott et al. 1966). Pour certaines souches, un test supplémentaire a été ajouté, la production d'acide à partir de saccharose (Lelliott et al. 1966).

Les souches d'Erwinia ont été identifiées au niveau de la sous-espèce selon les critères suivants: production d'oxydase, de catalase, de pectinase et d'indole, réduction du saccharose, production $\mathrm{d}^{\prime}$ acide à partir d' $\alpha$-méthyle glucoside, utilisation du glucose en conditions anaérobiques et croissance à $36^{\circ} \mathrm{C}$. Pour certaines souches, deux tests supplémentaires ont dû être faits (production $d^{\prime}$ acide à partir de tréhalose et utilisation du malonate). Tous ces tests ont été effectués selon les protocoles proposés par Dickey et Kelman (1988).

\section{Systèmes miniaturisés d'identification}

L'utilisation des systèmes API 20E [bioMérieux Canada inc., Saint-Laurent (Québec)], API NFT [bioMérieux Canada inc., Saint-Laurent (Québec)] et Biolog version 3.0 (Biolog inc., Hayward, Californie) a été faite en suivant les recommandations des manufacturiers. Les souches à tester provenaient d'une culture de 24 h sur du Triptic Soy Agar (Difco) pour les tests Biolog, et d'une culture de $24 \mathrm{~h}$ sur une gélose nutritive NGA (nutrient glucose agar) pour les tests API 20E et API NFT. Les incubations se faisaient à $30^{\circ} \mathrm{C}$ durant $24 \mathrm{~h}$ pour les tests API 20E et Biolog, et durant $48 \mathrm{~h}$ pour le test API NFT. Chaque souche de Pseudomonas a été testée trois fois sur les galeries API NFT et Biolog, alors que les souches d'Erwinia ont été testées trois fois sur les galeries API 20E et Biolog.

\section{Électrophorèse des protéines sécrétées}

Les protéines sécrétées ont été obtenues à partir de cultures liquides de $24 \mathrm{~h}$ qui se sont développées à $26^{\circ} \mathrm{C}$ dans le milieu M9 (Maniatis et al. 1982). Les cellules bactériennes ont été éliminées du surnageant par une centrifugation à $10500 \mathrm{~g}$ pendant $15 \mathrm{~min}$, suivie d'une filtration sur un filtre de cellulose avec des pores de $0,22 \mu \mathrm{m}$. Les protéines ont été concentrées en centrifugeant le surnageant dans un Centricon-10 (Amicon) à $4500 \mathrm{~g}$ pendant
60-90 min, ce qui permettait d'éliminer les composés de masse moléculaire inférieure à $10 \mathrm{kDa}$. Un dosage des protéines a alors été effectué par colorimétrie (Protein Assay, Biorad) afin d'analyser des quantités équivalentes de protéines pour chaque échantillon. Le gel de concentration contenait $4 \%$ de polyacrylamide $(\mathrm{pH} 6,7)$ et le gel de séparation contenait $12 \%$ de polyacrylamide $(\mathrm{pH} 8,9)$. L'électrophorèse a été effectuée selon la méthode de Laemmli (1970). Les gels ont été colorés au nitrate d'argent (Hames 1990). Les protéines ont été extraites au moins trois fois pour chaque souche bactérienne.

\section{RÉSULTATS}

\section{Caractérisation physiologique}

A l'aide de tests physiologiques, 54 souches de Pseudomonas et d'Erwinia, dont 44 ont été isolées au laboratoire de diagnostic, ont été caractérisées. L.es 44 souches isolées au laboratoire de diagnostic du MAPAO provenaient toutes de spécimens de plante différents; 19 de ces souches appartenaient au genre Erwinia et les 25 autres appartenaient au genre Pseudomonas (tableau 1). Les espèces les plus souvent isolées ont été I'E. carotovora ssp. carotovora (Jones) Bergey et al., le P. fluorescens IVb (Trevisan) Migula et le $P$. marginalis (Brown) Stevens. Nous avons également identifié des souches de $P$. viridiflava (Burkholder) Dowson et de $P$. syringae van Hall. Un seul test physiologique permettait de distinguer ces deux dernières espèces, soit la production d'acide à partir de saccharose, car le $P$. syringae produit de I'acide. De plus, des souches ont été identifiées comme E. carotovora ssp. atroseptica (van Hall) Dye. Les souches 166 et 169 produisaient de l'indole, ce qui caractérise l'E. chrysanthemi Burkholder, McFadden et Dimock. Cependant, nous avons classé ces deux souches dans le groupe des E. carotovorassp. carotovora, parce que les autres propriétés physiologiques de ces souches, dont la production d'acide à partir de tréhalose et l'incapacité à utiliser le malonate, les apparentaient à l'E. carotovora ssp. carotovora. 


\section{Identification des souches de Pseudomonas par API NFT et Biolog}

Le système Biolog (version 3) a identifié 9 des 12 souches de $P$. marginalis (selon les tests physiologiques) à au moins deux reprises comme étant le $P$. marginalis. Le système Biolog a fourni cette identification à 25 reprises sur les 36 tests réalisés. Pour 10 des 36 tests effectués, I'identification la plus probable a été $P$. corrugata; cependant la deuxième identification la plus probable était alors $P$. marginalis. Trois codes API NFT (1557555, 1757555 et $1557455)$ ont été fréquemment associés à ces souches; 11 des 12 souches ont obtenu, à deux ou trois reprises, un de ces trois codes les plus fréquents (tableau 2).

Plusieurs des souches de $P$. fluorescens (selon les tests physiologiques) n'ont pu être identifiées à l'espèce en utilisant le système Biolog. En effet, dans $40 \%$ des cas, le pourcentage de similarité entre nos souches et celles de la banque de données était inférieur à $50 \%$. Toutefois, en se basant sur l'identification la plus probable (que le pourcentage de similarité soit inférieur ou supérieur à $50 \%$ ), les identifications les plus fréquentes pour les 30 tests réalisés sont $P$. fluorescens à 11 reprises, $P$. marginalis à cinq reprises et Pseudomonas sp. à quatre reprises. Lors des essais avec les galeries API NFT, 24 codes différents ont été obtenus pour les 30 tests réalisés (tableau 2).

Il existe une excellente correspondance entre l'utilisation des tests physiologiques et I'utilisation du système Biolog pour identifier le $P$. viridiflava. Trois des quatre souches de $P$. viridiflava ont été identifiées comme telles, et ce, à trois reprises. Seule la souche 78 n'a été identifiée qu'à une seule reprise comme $P$. viridiflava. Le système Biolog ne réussit cependant pas à identifier les souches de $P$. syringae. Trois principaux codes API NFT étaient associés au $P$. viridiflava $(0457451,0457441$ et 0447451$)$. Deux codes principauxétaient associés au $P$. syringae (0477541 et 0477141) (tableau 2).

\section{Électrophorèse des protéines sécrétées par les souches de Pseudomonas}

Malgré le fait que les souches de Pseudomonas se soient développées en milieu minimal, un nombre relativement important de protéines ont été sécrétées dans le milieu de culture. De 6 à 18 protéines sécrétées ont été décelées dans les milieux de culture (fig. 1).

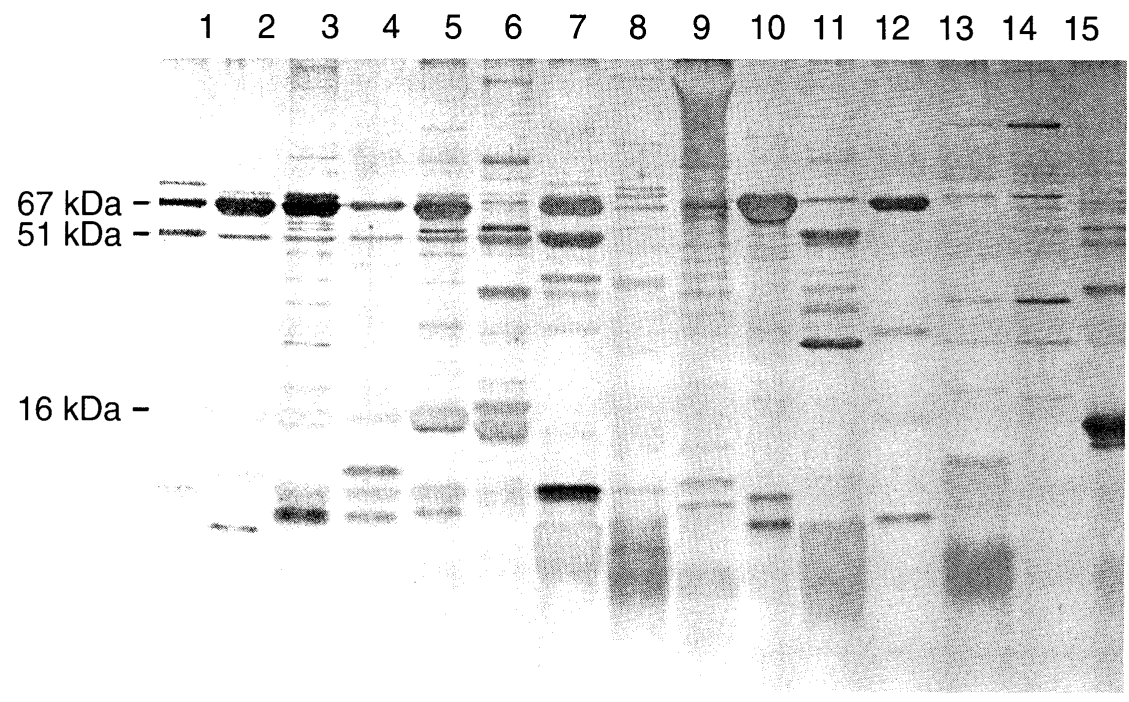

Figure 1. Électrophorèse des protéines sécrétées par des souches de Pseudomonas causant la pourriture molle. $P$. marginalis: puits 1-5, souches 115, 101, 95, 94 et 51, respectivement; $P$. fluorescens: puits 6-12, souches 296, 142, 129, 120, 114, 110 et 57, respectivement; $P$. syringae: puits 13 et 14, souches 135 et 97 , respectivement; $P$. viridiflava: puits 15 , souche 58 . Les chiffres à gauche de la figure sont des indicateurs de masse moléculaire. 
Tableau 2. Identification des souches de Pseudomonas par caractérisation physiologique et par les systèmes API NFT et Biolog version $\mathbf{3 . 0}$

\begin{tabular}{|c|c|c|c|c|}
\hline \multirow{2}{*}{$\begin{array}{l}\text { Identification par des } \\
\text { tests physiologiques }\end{array}$} & \multicolumn{2}{|c|}{ API NFT } & \multicolumn{2}{|l|}{ Biolog } \\
\hline & code & fréquence ${ }^{a}$ & identification $^{\mathrm{b}}$ & fréquence $^{c}$ \\
\hline P. marginalis & $\begin{array}{l}1557555 \\
1757555 \\
1557455 \\
0557455 \\
0157555 \\
0157455 \\
0157557 \\
1547455 \\
1555455 \\
1557755 \\
1447475\end{array}$ & $\begin{array}{r}13 / 36 \\
8 / 36 \\
5 / 36 \\
2 / 36 \\
2 / 36 \\
1 / 36 \\
1 / 36 \\
1 / 36 \\
1 / 36 \\
1 / 36 \\
1 / 36\end{array}$ & $\begin{array}{l}P . \text { marginalis } \\
P . \text { corrugata } \\
P . \text { fluorescens }\end{array}$ & $\begin{array}{r}25 / 36 \\
10 / 36 \\
1 / 36\end{array}$ \\
\hline P. viridiflava & $\begin{array}{l}0457451 \\
0457441 \\
0447451 \\
0450400 \\
0457551\end{array}$ & $\begin{array}{l}6 / 12 \\
2 / 12 \\
2 / 12 \\
1 / 12 \\
1 / 12\end{array}$ & $\begin{array}{l}\text { P. viridiflava } \\
P . \text { syringae } \\
\text { Aucune identification }\end{array}$ & $\begin{array}{r}10 / 12 \\
1 / 12 \\
1 / 12\end{array}$ \\
\hline$P$. syringae & $\begin{array}{l}0477541 \\
0477141 \\
0477551 \\
0477553 \\
0477573\end{array}$ & $\begin{array}{l}6 / 12 \\
3 / 12 \\
1 / 12 \\
1 / 12 \\
1 / 12\end{array}$ & Aucune identification & $12 / 12$ \\
\hline$P$. fluorescens & $\begin{array}{l}0157555 \\
0157557 \\
0157577 \\
0557455 \\
0157455 \\
0750457 \\
0150457 \\
0155457 \\
5557577 \\
1056557 \\
1042445 \\
1142445 \\
0342757 \\
0040457 \\
0041457 \\
5757555 \\
7657555 \\
7275555 \\
0762757 \\
0463757 \\
0663757 \\
1557555 \\
1757555 \\
1557557\end{array}$ & $\begin{array}{l}5 / 30 \\
2 / 30 \\
2 / 30 \\
1 / 30 \\
1 / 30 \\
1 / 30 \\
1 / 30 \\
1 / 30 \\
1 / 30 \\
1 / 30 \\
1 / 30 \\
1 / 30 \\
1 / 30 \\
1 / 30 \\
1 / 30 \\
1 / 30 \\
1 / 30 \\
1 / 30 \\
1 / 30 \\
1 / 30 \\
1 / 30 \\
1 / 30 \\
1 / 30 \\
1 / 30\end{array}$ & $\begin{array}{l}\text { P. fluorescens } \\
P . \text { marginalis } \\
\text { Pseudomonas sp. } \\
P . \text { corrugata } \\
P . \text { putida } \\
\text { Aucune identification }\end{array}$ & $\begin{array}{r}11 / 30 \\
5 / 30 \\
4 / 30 \\
1 / 30 \\
1 / 30 \\
8 / 30\end{array}$ \\
\hline
\end{tabular}

\footnotetext{
${ }^{a}$ Nombre de fois que le code a été obtenu/nombre de tests réalisés avec les souches d'une même espèce.

b Le système identifie une souche à l'espèce lorsque le taux de similarité est supérieur à $50 \%$.

c Nombre de fois que l'identification a été obtenue/nombre de tests réalisés avec les souches d'une même espèce.
} 
Certaines caractéristiques du profil électrophorétique des différentes espèces de Pseudomonas ont été relevées. Trois bandes majeures étaient habituellement présentes dans le surnageant des souches de $P$. marginalis. La masse moléculaire des trois protéines était approximativement de 67, 51 et $16 \mathrm{kDa}$. Une seule souche faisait exception: la souche 114, chez qui seule la protéine de $67 \mathrm{kDa}$ était présente.

Le profil protéique des souches de $P$. fluorescens contenait également une protéine de $67 \mathrm{kDa}$ avec ou sans une protéine de $51 \mathrm{kDa}$ (fig. 1). La quantité de ces protéines sécrétées dans le milieu de culture variait sensiblement entre les différentes souches de $P$. fluorescens. Cependant, pour une souche donnée, ces protéines étaient toujours en quantité plus importante. La protéine de $16 \mathrm{kDa}$ ne se trouvait chez aucune souche, à l'exception de la souche 115. Une forte hétérogénéité était toutefois observée entre les souches de $P$. fluorescens. Aucune protéine majeure de $67 \mathrm{kDa}$ ou de $51 \mathrm{kDa}$ n'a été révélée dans le profil des souches de $P$. viridiflava et $P$. syringae (fig. 1).

\section{Identification des souches d'Erwinia par API 20E et Biolog}

Chez E. carotovora ssp. carotovora, 16 des 18 souches ont été identifiées comme telles à au moins deux reprises avec le système Biolog. Seulement trois souches $\mathrm{d}^{\prime} E$. carotovora ssp. carotovora ont été associées à l'E. carotovorassp. atroseptica et ceci ne s'est produit qu'à une occasion sur trois (tableau 3 ).

Seulement une souche d'E. carotovora ssp. atroseptica sur les six souches utilisées, a été identifiée comme telle à au moins deux reprises avec le système Biolog. Les autres souches ont été identifiées comme $E$. carotovora ssp. carotovora, et dans certains cas, le système ne permettait pas de déterminer de quelle sous-espèce il s'agissait (tableau 3 ).

Le système API $20 \mathrm{E}$ a fourni trois principaux codes $(1207373,1207173$ et 1205173) pour 40 des 54 essais effectués avec les souches d'E. carotovora ssp. carotovora. L'un destrois principauxcodes a été associé à au moins deux reprises pour 14 des 18 souches d'E. carotovora ssp. carotovora. Un seul code API 20E

Tableau 3. Identification des souches d'Erwinia par caractérisation physiologique et par les systèmes API $20 \mathrm{E}$ et Biolog version 3.0

\begin{tabular}{|c|c|c|c|c|}
\hline \multirow{2}{*}{$\begin{array}{l}\text { Identification par des } \\
\text { tests physiologiques }\end{array}$} & \multicolumn{2}{|c|}{ API 20E } & \multicolumn{2}{|l|}{ Biolog } \\
\hline & code & fréquence $^{\mathrm{a}}$ & identification $^{b}$ & fréquence $^{c}$ \\
\hline \multirow[t]{12}{*}{ E. carotovora ssp. carotovora } & 1207373 & $16 / 54$ & E. carotovora ssp. carotovora & $45 / 54$ \\
\hline & 1207173 & $15 / 54$ & E. carotovora ssp. atroseptica & $3 / 54$ \\
\hline & 1205173 & $9 / 54$ & Erwinia sp. & $2 / 54$ \\
\hline & 1205363 & $3 / 54$ & Enterobacter agglomerans & $1 / 54$ \\
\hline & 1205373 & $2 / 54$ & Aucune identification & $3 / 54$ \\
\hline & 1245773 & $2 / 54$ & & \\
\hline & 1007173 & $2 / 54$ & & \\
\hline & 1207573 & $1 / 54$ & & \\
\hline & 1207333 & $1 / 54$ & & \\
\hline & 1205573 & $1 / 54$ & & \\
\hline & 1245173 & $1 / 54$ & & \\
\hline & 1005173 & $1 / 54$ & & \\
\hline \multirow[t]{3}{*}{ E. carotovora ssp. atroseptica } & 1205173 & $17 / 18$ & E. carotovora ssp. carotovora & $9 / 18$ \\
\hline & 1207173 & $1 / 18$ & Erwinia sp. & $6 / 18$ \\
\hline & & & E. carotovora ssp. atroseptica & $3 / 18$ \\
\hline
\end{tabular}


(1205173) a été associé aux souches d'E. carotovora ssp. atroseptica. Ce code a été rarement associé à l'E. carotovora ssp. carotovora (tableau 3).

\section{Electrophorèse des protéines sécrétées par les souches d'Erwinia} Tout comme pour les Pseudomonas, de nombreuses protéines sont sécrétées à l'extérieur des cellules lorsque les souches d'Erwinia carotovora croissent dans le milieu minimal M9. La présence de 8 à 22 protéines a été révélée par une coloration au nitrate d'argent. Les patrons électrophorétiques variaient fortement entre les souches (fig. 2).

\section{DISCUSSION}

La classification des Pseudomonas fluorescents est toujours controversée. Dans la dernière édition du Bergey's Manual of Systematic Bacteriology (Palleroni 1984), ces bactéries sont divisées en deux groupes selon leur production d'arginine déshydrolase. Parmi les bactéries productrices d'arginine déshydrolase, on compte cinq espèces, dont le
$P$. fluorescens. L'espèce $P$. fluorescens se divise en cinq biovars. Les bactéries communément appelées $P$. marginalispar les phytopathologistes appartiennent en fait au biovar Il du P. fluorescens. Toutefois, dans ce travail, nous référerons à ces bactéries sous le nom de $P$. marginalis. Parmi les espèces ne produisant pas $d$ 'arginine déshydrolase, nous comptons trois espèces dont le $P$. syringae et le $P$. viridiflava.

Aucune des techniques utilisées n'est un outil de diagnostic parfait. Même les tests classiques de caractérisation physiologique peuvent s'avérer imparfaits. Par exemple, la souche 115, qui a été identifiée comme $P$. fluorescens par cette méthode, semble plutôt appartenir à $I^{\prime}$ espèce $P$. marginalis si I'on se fie à toutes les autres méthodes d'identification. De même, la souche 114 de $P$. marginalis devrait être classée comme P. fluorescens selon les autres méthodes d'identification.

Les souches de $P$. marginalis semblent former une population relativement homogène. Le système d'identification de Biolog permet de reconnaître la

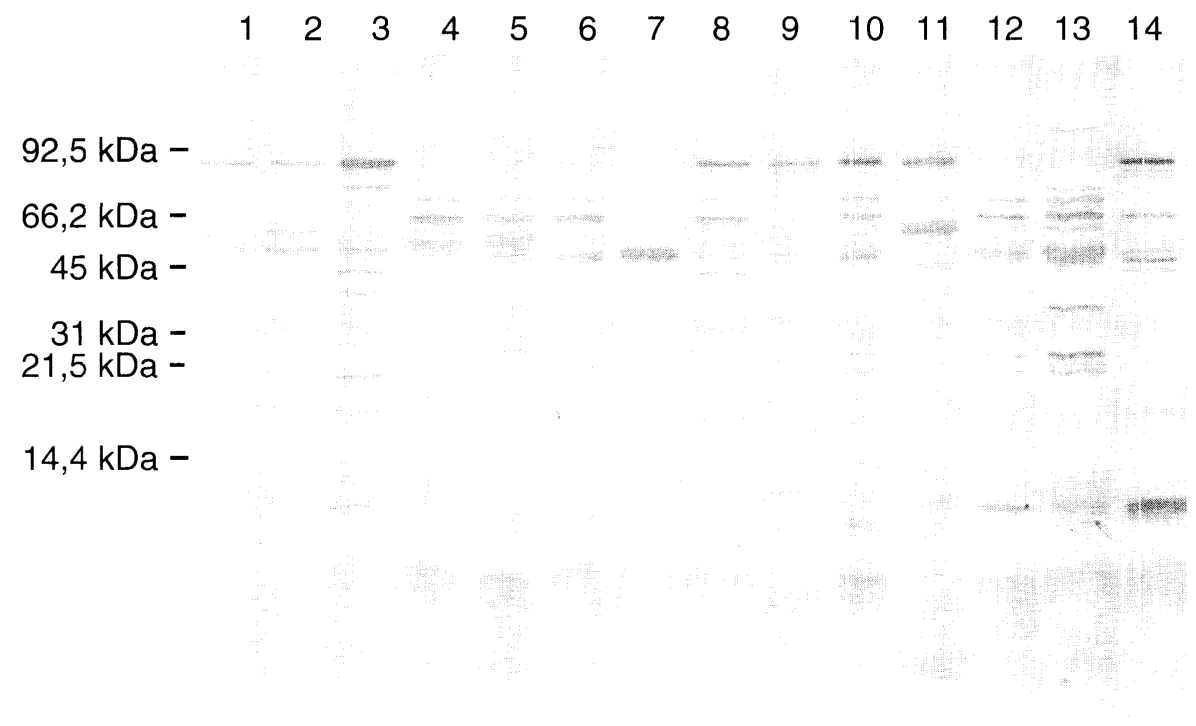

Figure 2. Électrophorèse des protéines sécrétées par des souches d'Erwinia causant la pourriture molle. E. carotovora ssp. atroseptica: puits 1-3, souches 159, 157 et 22, respectivement; E. carotovora ssp. carotovora: puits $4-14$, souches 169, 166, 182, 172, 163, 162, 153, 138, 130, 74 et 13 , respectivement. Les chiffres à gauche de la figure sont des indicateurs de masse moléculaire. 
majorité des souches de cette espèce. De plus, trois codes API NFT (1557555, 1757555 et 1557455), ne différant entre eux qu'à une ou deux positions, étaient associés aux souches de $P$. marginalis. Toutes les souches de $P$. marginalis se caractérisent aussi par la présence, dans leur profil protéique, d'une quantité importante de trois protéines de 67,51 et $16 \mathrm{kDa}$. La proximité taxonomique entre le $P$. marginalis et le $P$. fluorescens est confirmée par l'analyse des profils des protéines sécrétées. Tout comme pour le $P$. marginalis et contrairement au $P$. syringae et au $P$. viridiflava, des quantités importantes de protéines de $67 \mathrm{kDa}$ ou de $51 \mathrm{kDa}$ (ou de ces deux protéines) sont sécrétées par toutes les souches de $P$. fluorescens.

En dépit de la proximité taxonomique entre le $P$. viridiflava et le $P$. syringae, les souches de ces espèces peuvent être différenciées par le système API NFT. En effet, bien que les codes API NFT associés au deux espèces se ressemblent beaucoup, le troisième chiffre du code est toujours le 7 pour les souches de $P$. syringae, alors qu'il est le 4 ou le 5 pour les souches de $P$. viridiflava. Le système Biolog ne permet pas d'identifier le $P$. syringae, bien qu'il reconnaisse le $P$. viridiflava pour 10 des 12 tests réalisés (83\%). Jones et al. (1993) obtiennent un résultat différent pour le $P$. syringae puisque $100 \%$ des souches de cette espèce furent identifiées avec précision avec le système Biolog. Cependant, les résultats de Jones et al. (1993) pour I'identification de $P$. viridiflava sont similaires à ceux de notre étude, puisque $87 \%$ des souches de cette espèce sont reconnues avec le système Biolog.

La variabilité observée entre les souches de $P$. fluorescens, qui a conduit à la division de l'espèce en de nombreux biovars, s'observe également dans la population des souches responsables de la pourriture molle. Les nombreux codes API NFT, associés aux souches de $P$. fluorescens étudiées dans ce travail, et I'hétérogénéité des identificationsfournies par le système Biolog pour les souches de $P$. fluorescens, montrent bien la variabilité importante des souches de cette espèce. L'hétérogénéité observée dans ce groupe de bactéries rend donc difficile l'emploi des systèmes miniaturisés comme outil de diagnostic. Cependant, nos résultats $d^{\prime}$ identification de $P$. fluorescens avec le système Biolog diffèrent de ceux d'autres études. En effet, Jones et al. (1993) et Klingler et al. (1992) rapportent que le $P$. fluorescens fut identifié avec précision dans $100 \%$ des cas avec le système Biolog.

L'électrophorèse des protéines sécrétées apparaît la technique la plus fiable pour différencier le $P$. fluorescens du $P$. marginalis. Trois protéines de 67, 51 et $16 \mathrm{kDa}$ sont associées au P. marginalis alors que seules les protéines de 67 et $51 \mathrm{kDa}$ étaient retrouvées dans le surnageant des souches de $P$. fluorescens. Quant aux souches de $P$. viridiflava et de $P$. syringae, elles semblent se caractériser par l'absence des protéines de 67 et $51 \mathrm{kDa}$. Une analyse d'un nombre plus élevé de souches de Pseudomonas serait toutefois nécessaire pour confirmerl'utilité de l'électrophorèse des protéines sécrétées comme outil d'identification des Pseudomonas.

Les systèmes miniaturisés d'identification (API 20E et Biolog) se montrent très efficaces pour reconnaître I'E. carotovora ssp. carotovora. Avec le système Biolog, nous avons toutefois certaines difficultés à reconnaître les souches $d^{\prime} E$. carotovora ssp. atroseptica. Tout comme Jones et al. (1993), nos résultats permettent d'identifier avec précision l'E. carotovora sans toutefois différencier les sous-espèces. Le système API $20 E$ attribue un code unique à I'E. carotovora ssp. atroseptica. Ce code est parfois rencontré avec l'E. carotovora ssp. carotovora. Dans une étude antérieure, Mergaert et al. (1984) avaient associé cinq codes API 20E différents aux souches d'E. carotovora ssp. carotovora. Ces codes ont été associés pour 25 des 54 tests réalisés avec les souches d'E. carotovora ssp. carotovora isolées au Québec.

Plusieurs protéines sont sécrétées par les souches d'E. carotovora; toutefois, trop de variations sont observées dans les patrons protéiques pour que ces derniers soient utiles à des fins de diagnostic. La détection de protéines cellulaires spécifiques, telles les pectinases, cellulases, protéases, etc., pourrait éventuellement permettre de reconnaître les deux sousespèces. Dans un travail récent, la 
production de pectinases par l'E. chrysanthemi a été détectée in planta (Beaulieu etal. 1993). La technique consiste à récupérer les fluides des tissus macérés et à y séparer les différentes pectinases par électrofocalisation. II serait maintenant intéressant de déterminer si une technique aussi simple pourrait être adoptée pour des fins de diagnostic.

\section{REMERCIEMENTS}

Nous remercions C. Goyer pour ses commentaires sur le manuscrit, P.D. Hildebrand et J.F. Chauveau pour le don de souches bactériennes, et $B$. Drouin pour son aide dans le montage des figures.

\section{RÉFÉRENCES}

Beaulieu, C., M. Boccara et F. Van Gijsegem. 1993. Pathogenic behavior of pectinasedefective Erwinia chrysanthemi mutants on different plants. Mol. Plant-Microbe Interact. 6: 197-202.

Boccara, M., R. Vedel, D. Lalo, M.-H. Lebrun et J.F. Lafay. 1991. Genetic diversity and host range in strains of Erwinia chrysanthemi. Mol. Plant-Microbe Interact. 4 : 293-299.

Cappellini, R.A., M.J. Ceponis et G.W. Lightner. 1987. Disorders in celery and carrot shipments to the New York market, 19721985. Plant Dis. 71: 1054-1057.

Ceponis, M.J., R.A. Cappellini et G.W. Lightner. 1985. Disorders in crisphead lettuce shipments to the New York market, 19721984. Plant Dis. 69: 1016-1020.

Darrasse, A., S. Priou, A. Kotoujansky et Y. Bertheau. 1994. $P C R$ and restriction fragment length polymorphism of a pel gene as a tool to identify Erwinia carotovora in relation to potato diseases. Appl. Environ. Microbiol. 60: 1437-1443.

Dickey, R.S. et A. Kelman. 1988. Erwinia. Pages 44-59 in N.W. Schaad (réd.), Laboratory guide for identification of plant pathogenic bacteria. APS Press, St. Paul, Minnesota.

Dickey, R.S., C.H. Zumoff et J.K. Uyemoto. 1984. Erwinia chrysanthemi: serological relationships among strains from several hosts. Phytopathology 74 : 1388-1394.

Gitaitis, R.D., R.E. Baird, R.W. Beaver, D.R. Sumner, J.D. Gay et D.A. Smittle. 1991. Bacterial blight of sweet onion caused by Pseudomonas viridiflava in Vidalia, Georgia. Plant Dis. 75: 1180-1182.
Hames, B.D. 1990. Analysis of gels following electrophoresis. Pages 51-96 in B.D. Hames et D. Rickwood (réds.), Gel electrophoresis of proteins: a pratical approach. IRL. Press, New York.

Hildebrand, D.C. 1971. Pectate and pectin gels for differentiation of Pseudomonas sp. and other bacterial plant pathogens. Phytopathology 61: 1430-1436.

Hildebrand, P.D. 1989. Surfactant-like characteristics and identity of bacteria associated with broccoli head rot in Atlantic Canada. Can. J. Plant Pathol. 11: 205-214.

Jones, J.B., A.R. Chase et G.K. Harris. 1993. Evaluation of the Biolog GN MicroPlate system for identification of some plantpathogenic bacteria. Plant Dis. 77: 553558.

King, E.O., M.K. Ward et D.E. Raney. 1954. Two simple media for the demonstration of pyocyanin and fluorescein. J. Lab. Clin. Med. 44: 301-307.

Klingler, J.M., R.P. Stowe, D.C. Obenhuber, T.O. Groves, S.K. Mishra et D.L. Pierson. 1992. Evaluation of the Biolog automated microbial identification system. Appl. Environ. Microbiol. 58: 2089-2092.

Laemmli, U.K. 1970. Cleavage of structural proteins during the assembly of the head of bacteriophage T4. Nature (Lond.) 227 : 680-686.

Lelliott, R.A., E. Billing et A.C. Hayward. 1966. A determinative scheme for the fluorescent plant pathogenic pseudomonads. J. Appl. Bacteriol. 29 : 470-489.

Liao, C.H. et J.M. Wells. 1987a. Association of pectolytic strains of Xanthomonas campestris with soft rots of fruits and vegetable at retail markets. Phytopathology 77: 418-422.

Liao, C.H. et J.M. Wells. 1987b. Diversity of pectolytic, fluorescent pseudomonads causing soft rots of fresh vegetables at produce markets. Phytopathology 77 : 673677.

Lund, B.M. 1983. Bacterial spoliage. Pages 219-257 in C. Dennis (réd.), Post-harvest pathology of fruits and vegetables. Acad. Press, Londres.

Maniatis, T., E.F. Ffristsh et J. Sambrook. 1982. Molecular cloning: a laboratory manual. Cold Spring Harbor Lab., Cold Spring Harbor, New York.

Mergaert, J., L. Verdonck, K. Kersters, J. Swings, J.-M. Boeufgras et J. De Ley. 1984. Numerical taxonomy of Erwinia species using API systems. J. Gen. Microbiol. 130: 1893-1910. 
Miller, T.D. et M.N. Schroth. 1972. Monitoring the epiphytic population of Erwinia amylovora on pear with a selective medium. Phytopathology 62: 1175-1182.

Palleroni, N.J. 1984. Family I. Pseudomonadaceae Winslow, Broadhurst, Buchanan, Krumwiede, Rogers and Smith 1917, 555'. Pages 141-218 in N.R. Krieg and J.G. Holt (réd.), Bergey's manual of systematic bacteriology, Volume 1. Williams and Wilkins, Baltimore.

Paradis, E., C. Goyer, N.C. Hodge, R. Hogue, R.E. Stall et C. Beaulieu. 1994. Fatty acid and protein profiles of Streptomyces scabies strains isolated in Eastern Canada. Int. J. Syst. Bacteriol. 44: 561-564.

Pieczarka, D.J. et J.W. Lorbeer. 1975. Microorganisms associated with bottom rot of lettuce grown on organic soil in New York State. Phytopathology 65: 16-21.
Samson, R., F. Poutier, M. Sailly et B. Jouan. 1987. Caractérisation des Erwinia chrysanthemi isolées de Solanum tuberosum et d'autres plantes-hôtes selon les biovars et sérogroupes. Bulletin OEPP (Organ. eur. méditerr. prot. plantes) 17: 11-16.

Van Zyl, E. et P.L. Steyn. 1990. Differentiation of phytopathogenic Pseudomonas and Xanthomonas species and pathovars by numerical taxonomy and protein gel electrophoregrams. Syst. Appl. Microbiol. 13: 60-71.

Ward, L.J. et S.H. De Boer. 1994. Specific detection of Erwinia carotovora subsp. atroseptica with a digoxigenin-labeled DNA probe. Phytopathology 84 : 180-186. 